\title{
Lifetime Analysis of Super Capacitor for Many Power Electronics Applications
}

\author{
Rajib Sarkar Rajan, Md. Moshiur Rahman \\ Department of Electrical and Electronic Engineering, University of Asia Pacific, Bangladesh
}

\begin{abstract}
Supercapacitoris an electrochemical energy storage device with a high power and energy density. It acts as an energy buffer or energy sources due to their high power density compared to other charge storing devices. To improve the performance, reliability and using this device more conveniently lifetime is a important feature. So in this paper we analyze lifetime of supercapacitor depends on temperature and voltage factor because lifetime of supercapacitors depends mainly on the capacitor temperature and the voltage applied. Moreover to prove validity of analysis simulation results are presented. By using this analysis it is easier to select supercapacitor in many power electronics application.
\end{abstract}

Keywords :Supercapacitor, Expected Lifetime, Temperature Factor, Voltage Factor, Electric Double Layer Capacitor (EDLC).

\section{INTRODUCTION}

With the changing global landscape, energy has become a primary focus for all the countries. As a result world scientist community shows their great interest in developing and refining more efficient energy storage devices.

One such device, the supercapacitors, has matured significantly over the last decade and emerged with the potential to facilitate major advances in energy storage. Conventional batteries and capacitors are widely used for energy storing. Although conventional batteries have very high energy density but the lower cyclic lifetime and low power density restricts their application in high power system [1]. The instantaneous discharging behavior and low energy density reduce the reliability of the conventional capacitors in many applications. Compared with batteries and conventional capacitors, supercapacitors are more preferable due to their high-efficiency, lightweight, larger current density, wider working temperature range, less maintenance and environmental friendly [1]. Supercapacitors also are distinguished from other storage devices by a much longer lifetime. Lifetime of supercapacitor is very important to select this device more cost effectively for many applications. In this paper we observe and analyze temperature and voltage effect on life time. Analysis of lifetime was validated with simulation result .In this paper we first introduce scope and energy storing principle of supercapacitor in section II. In section III we have analyzed lifetime of supercapacitor based on temperature and voltage effect .Conclusion of this study is given in section IV.

\section{SUPERCAPCITOR GENERALS}

\subsection{Scope of supercapacitor}

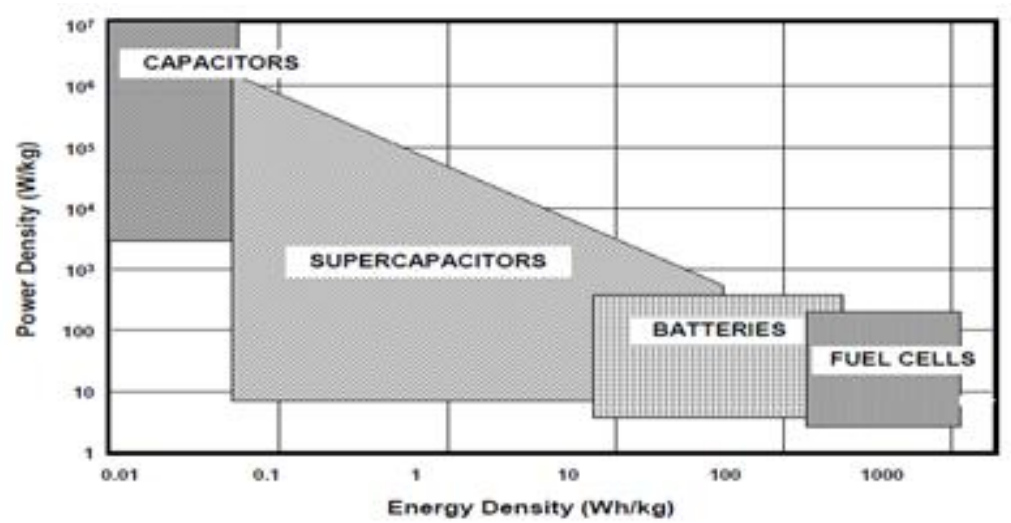

Fig.1. Performance of different charge storage device

Supercapacitors, also known as ultra capacitors or electric double layer capacitors (EDLC), are electrical energy storage devices, which offer high power density, extremely high cycling capability and mechanical robustness. Due to their electrical performance, supercapacitors have a high potential to be used in industrial applications 
such as electric vehicles, fuel cell vehicles, computer systems, welder, inverters, automobile regenerative braking systems, power supplies etc. Moreover, supercapacitors can be used for desalting water based on periodic sorption and desorption of ions on the extensive surface of porous electrode based on its capacitive properties [2], [3].To observe advantage of supercapacitor over other charge storage devices Ragone plot is used. In Fig. 1 it is seen that supercapacitors occupy a region between conventional capacitors and batteries.

\subsection{Energy storage principle}

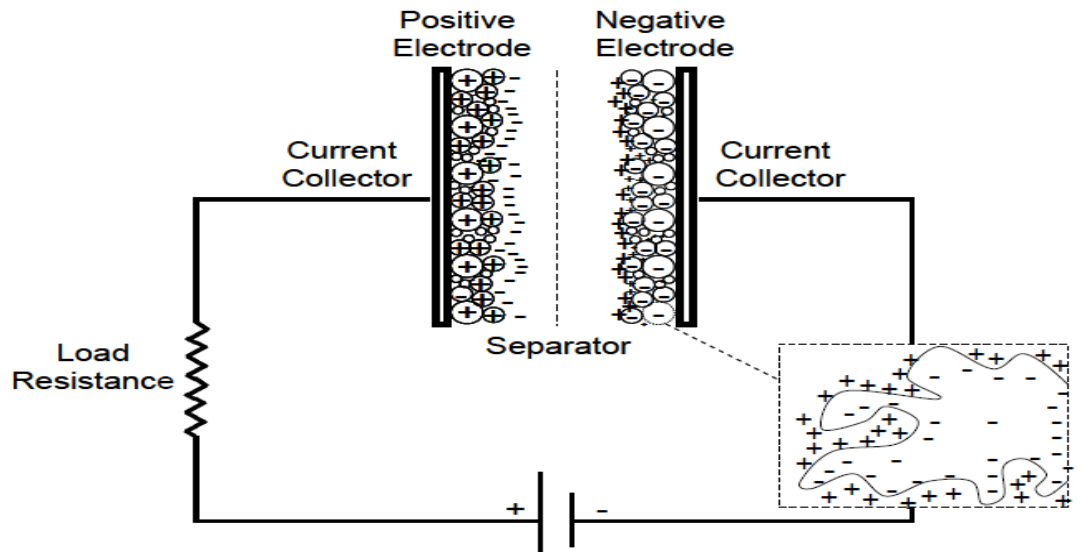

Fig.2. Charge storage principle of supercapacitor

Supercapacitors consist of two carbon based electrodes separated by a dielectric material. The dielectric not only separates the electrodes but also has electrical properties that affect the performance of a supercapacitor. Actually here separator acts as an insulator which prevents physical contact of electrodes but allows ion transfer between them [4].Supercapacitors also store charge electro statically, and there is no transfer of charge between electrode and electrolyte. In response to the applied voltage, an electric field is created in the electrolyte which causes polarization in the electrolyte. Due to the natural attraction of unlike charges, ions in the electrolyte diffuse across the separator into the pores of the electrode of opposite charge. Thus, a double-layer of storing charge is produced at each electrode. As a result surface area is increased and decreases the distance between electrodes, which allow supercapacitor to achieve higher energy densities than conventional capacitors [5]-[7]. Electric energy was stored into the electrical double layer which was formed at the porous solid electrode/electrolyte interface [8]. Energy storing capacity of supercapacitor does not depend only on the specific surface area of electrode materials, but it also depends on the utilization rates of micro-hole of the porous electrode and the active materials in electrolyte [9].Different types of carbon materials used as an electrode such as activated carbon, carbon aero gels, carbon nanotubes and metal oxides. Instead of traditional dielectric material like ceramic, polymer films or aluminum oxide here $\mathrm{H} 2 \mathrm{SO} 4$ or $\mathrm{KOH}$ is used.

\section{LIFETIME ANALYSIS}

Supercapacitors has much longer lifetime than other charge storage devices. As there is no transfer of charge between electrolyte and electrode, there are no chemical or composition changes associated with nonFaradic processes. Except for those with polymer electrodes, its lifetimes depend mostly on the evaporation of the liquid electrolyte over time. This evaporation is a function of temperature and voltage applied. So to analyze lifetime of supercapacitor, temperature and voltage factor play a vital rule. In this paper we consider moisture factor equal to one.

\subsection{Expected lifetime}

Supercapacitor lifetime will be decreases with increasing temperature, applied voltage, humidity, current and backup time requirements. Considering these reality expected lifetime can be define

$$
E_{L}=\left(L_{R}\right) \cdot\left(T_{F}\right) \cdot\left(V_{F}\right) \cdot\left(M_{F}\right)
$$

Where $E_{L}, L_{R}, T_{F}, V_{F}, M_{F}$ are the expected lifetime, rated lifetime temperature factor, voltage factor and moisture factor respectively. 


\subsection{Proposed analysis}

According to "10-degrees-rule" lifetime of supercapacitor will be double for each 10 degree Celsius reduction of rated operating temperature. This is known as temperature factor which can be expressed as

$$
T_{F}=2^{\frac{\left(T_{R}-T_{O}\right)}{10}}
$$

In (2), $T_{R}$ for rated temperature and $T_{O}$ for operating temperature. From (2), we can see that lifetime will be maximum at $T_{O}=0$. But different appliances operating at different temperature where $T_{O}$ never be zero. So modified temperature factor is

$$
T_{F}=2^{\frac{\left(T_{R}-T_{A}\right)}{10}}
$$

Here $T_{A}$ for appliance operating temperature. And the effect on life extension is roughly proportional to the voltage decreasing. This relation can be defining as voltage factor.

$V_{F}=\frac{V_{R}}{V_{A}}$

Here in (4), $V_{R}$ for rated voltage and $V_{A}$ for applied voltage. According to (4) if we continuously decrease applied voltage, voltage factor will be increases as well as lifetime will be increases. But this is not practically visible. Because we can't reduce the voltage below the cut off voltage of the appliances where supercapacitor is used. So a modification is needed in voltage factor equation and that is

$V_{F}=\frac{V_{R}}{V_{C} \sim V_{R}}(5)$

In (5), $V_{C} \sim V_{R}$ represent the range between cut off to rated applied voltage. Considering all these modification and from (1), (3) and (5) expected lifetime of supercapacitor can be expressed as

$$
E_{L}=\left(L_{R}\right) \cdot\left(2^{\frac{\left(T_{R}-T_{A}\right)}{10}}\right) \cdot\left(\frac{V_{R}}{V_{C} \sim V_{R}}\right) \cdot\left(M_{F}\right)
$$

\section{VALIDITY OF THIS PROPOSED MODELING}

To prove validity of the analysis, at first we observe temperature and voltage factor effect on lifetime without modification. Here for simulation we used a supercapacitor sample EDLF105B5R5C

Table I. Parameters of EDLF105B5R5C

\begin{tabular}{|c|c|}
\hline Rated Voltage & $5.5 \mathrm{~V}$ \\
\hline Capacitance & $1 \mathrm{~F}$ \\
\hline Rated Temperature & $85^{\circ} \mathrm{C}$ \\
\hline Rated Lifetime & 1000 Hours \\
\hline
\end{tabular}

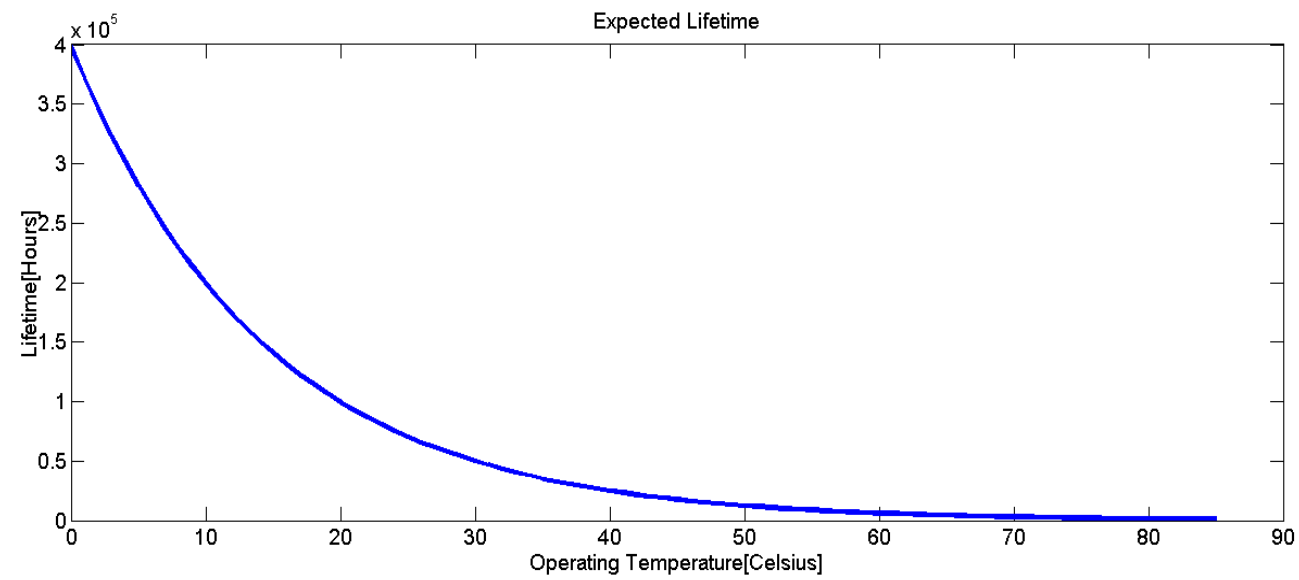

Fig.3.Expected lifetime based on temperature factor without modification

In Fig. 3 expected lifetime was illustrated based on temperature factor without modification, where lifetime is maximum at $0^{\circ} \mathrm{C}$ operating temperature. But this is not practical, because the appliances are not operates in $0^{0} \mathrm{C}$. Here voltage factor is 1.1 means supplied voltage is 5 volt and moisture factor is assume 1. 


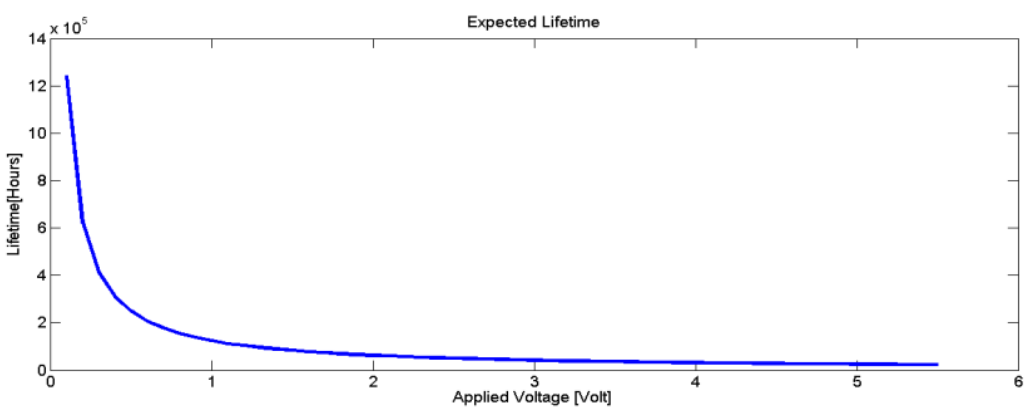

Fig.4. Expected lifetime based on voltage factor without modification

In Fig.4 expected lifetime was illustrated based on voltage factor without modification, where lifetime is maximum when applied voltage is zero. But this is not practical, because we can't reduce operating voltage below cut off voltage of appliances. Here temperature factor is 22.6 means operating temperature is $40^{\circ} \mathrm{C}$ and moisture factor is assume 1 . Now we consider a practical situation, where the sample is used in a circuit having an ambient temperature $40^{\circ} \mathrm{C}$ and cut off voltage $2 \mathrm{Vdc}$. In this situation modified lifetime equation (6) is needed to simulate actual lifetime.

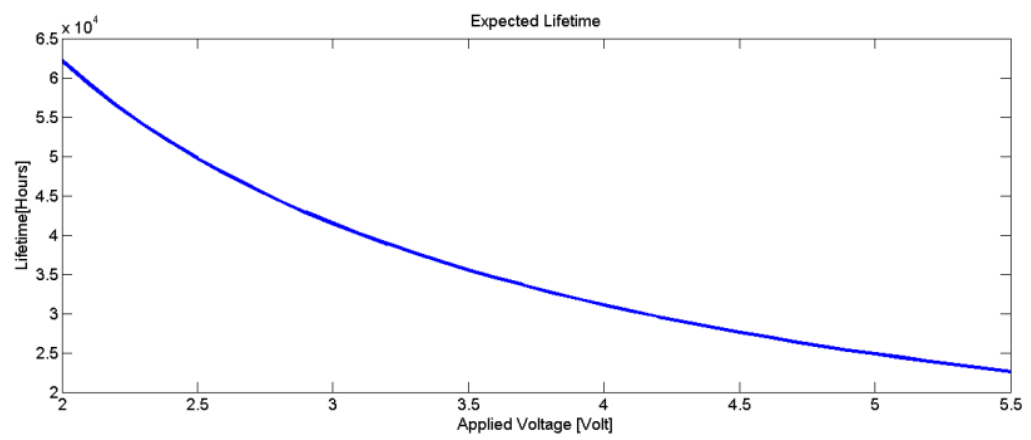

Fig.5. Expected lifetime based on voltage factor without modification

Fig.5 represents actual lifetime range of a supercapacitor sample which is used in a particular application, where lifetime is maximum at cut off voltage and minimum at rated voltage. Similar to this curve, it is possible to analyze actual lifetime of a supercapacitor in power electronics as well as other application.

\section{CONCLUSION}

Supercapacitor is a novel solution for energy storage because of its high power and energy density which is almost 10 to 20 times higher than conventional capacitors and batteries. Moreover it is environment friendly, less expensive, maintenance and hazard free All these qualities have driven the scientists and engineers worldwide to modeling supercapacitors for application in power electronics. To model this device lifetime analysis is a major concern. So our effort in analyzing lifetime of supercapacitor is also participating with the world scientists and engineers in an effort to develop the model of a supercapacitor. In this paper some modification are given in expected lifetime equation to makes it practical. This unique analysis can be used to find out lifetime of a supercapacitor in many power electronics application.

\section{REFERENCES}

[1] Ran Niu and Hai Yang, "Modeling and Identification of Electric Double Layer Supercapacitors", ICRA Communication ( IEEE Conference), pp. $1-4,2011$.

[2] A.M. Johnson and J. Newman, "Desalting by Means of Porous Carbon Electrodes", J. Electrochem.Soe.,vol. 118, pp. 510-517, 1971.

[3] Y. Oren, "Capacitive deionization (CDI) for desalination and water treatment - past, present and future (a review)", Desalination, vol. 228, pp. 10-29, 2008.

[4] O.H. LeBlanc, Mathematics of Ultracapacitors, GE Global Research, Technical Report, June 1993.

[5] Conway, B. E. (1999). Electrochemical Supercapacitors : Scientific Fundamentals and technological Applications. New York, Kluwer-Plenum.

[6] Burke, A. (2000). "Ultracapacitors: why, how, and where is the technology." Journal of Power Sources 91(1): 37-50.

[7] Kotz, R. and M. Carlen (2000). "Principles and applications of electrochemical capacitors."ElectrochimicaActa 45(15-16): 24832498.

[8] Z. Li, C. Jie, "An impedance-based approach to predict the state-of charge for carbon-based supercapacitors", Microelectronic Engineering, vol. 85, pp. 1549-1554, 2008

F.Belhachemi, S. Rael and B. Davat, "A physical based model of power electric double-layer supercapacitors", Industrial Application Conference, vol. 5, pp. 3069-3076, 2000. 